\title{
Quantitative evaluation of aerosol generation during manual facemask ventilation
}

\author{
A. J. Shrimpton, ${ }^{1}$ (D) J. M. Brown, ${ }^{2}$ F. K. A. Gregson, ${ }^{3}$ (D) T. M. Cook, ${ }^{4}$ (D) D. A. Scott, ${ }^{5}$ \\ F. McGain, ${ }^{6}$ (D) R. S. Humphries, ${ }^{7}$ R. S. Dhillon, ${ }^{8}$ (D) J. P. Reid, ${ }^{9}$ (iD F. Hamilton, ${ }^{10}$ (D) \\ B. R. Bzdek ${ }^{11}$ (iD) and A. E. Pickering, ${ }^{12}$ (i) on behalf of the AERATOR study group*
}

\begin{abstract}
1 Doctoral Fellow, 12 Professor, Anaesthesia, Pain and Critical Care Sciences, School of Physiology, Pharmacology and Neuroscience, 10 Doctoral Fellow, Department of Population Health Sciences, University of Bristol, Bristol, UK 2 Consultant, Department of Anaesthesia and Intensive Care Medicine, North Bristol NHS Trust, Bristol, UK 3 Visiting Research Associate, 9 Professor, 11 Research Fellow, School of Chemistry, University of Bristol, Bristol, UK 4 Honorary Professor and Consultant, Department of Anaesthesia and Intensive Care Medicine, Royal United Hospital NHS Trust, Bath, UK

5 Professor, Department of Critical Care, University of Melbourne; St. Vincent's Hospital Melbourne, Australia 6 Anaesthetist and Intensive Care Physician, Western Health, Footscray, Victoria, Australia

7 Research Scientist, Climate Science Centre, CSIRO Oceans and Atmosphere, Aspendale, Victoria, Australia 8 Consultant, Department of Neurosurgery, St Vincent's Hospital Melbourne, Fitzroy, Victoria, Australia
\end{abstract}

\begin{abstract}
Summary
Manual facemask ventilation, a core component of elective and emergency airway management, is classified as an aerosol-generating procedure. This designation is based on one epidemiological study suggesting an association between facemask ventilation and transmission during the SARS-CoV-1 outbreak in 2003. There is no direct evidence to indicate whether facemask ventilation is a high-risk procedure for aerosol generation. We conducted aerosol monitoring during routine facemask ventilation and facemask ventilation with an intentionally generated leak in anaesthetised patients. Recordings were made in ultraclean operating theatres and compared against the aerosol generated by tidal breathing and cough manoeuvres. Respiratory aerosol from tidal breathing in 11 patients was reliably detected above the very low background particle concentrations with median [IQR (range)] particle counts of 191 (77-486 [4-1313]) and 2 (1-5 [0-13]) particles. I $^{-1}$, respectively, $\mathrm{p}=0.002$. The median (IQR [range]) aerosol concentration detected during facemask ventilation without a leak (3 (0-9 [0-43]) particles..$\left.^{-1}\right)$ and with an intentional leak (11 (7-26 [1-62]) particles..$\left.^{-1}\right)$ was 64-fold $(p=0.001)$ and 17 -fold $(p=0.002)$ lower than that of tidal breathing, respectively. Median (IQR [range]) peak particle concentration during facemask ventilation both without a leak (60 (0-60 [0-120]) particles..$\left.^{-1}\right)$ and with a leak (120 (60-180 [60-480]) particles. $\left.\left.\right|^{-1}\right)$ were 20-fold $(p=0.002)$ and 10-fold $(0.001)$ lower than a cough (1260 (800-3242 [100-3682]) particles. ${ }^{-1}$ ), respectively. This study demonstrates that facemask ventilation, even when performed with an intentional leak, does not generate high levels of bioaerosol. On the basis of this evidence, we argue facemask ventilation should not be considered an aerosol-generating procedure.
\end{abstract}

Correspondence to: A. J. Shrimpton

Email: andy.shrimpton@bristol.ac.uk

Accepted: 21 September 2021

Keywords: aerosol-generating procedure; COVID-19; facemask ventilation; manual ventilation; SARS-CoV-2

*See Appendix 1

Twitter: @_andyshrimp; @drjulesbrown; @doctimcook; @scottdav44; @ForbesMcGain; @gushamilton; @TonyPi314 


\section{Introduction}

The COVID-19 pandemic continues to place unprecedented demands on healthcare globally. The use of airborne personal protective equipment (PPE) has been reserved largely for healthcare workers undertaking medical procedures deemed to be aerosol generating [1-3]. These procedures are presumed to generate as much or higher levels of bioaerosols from the respiratory tract than coughing and consequently carry an increased risk of viral transmission. The evidence for these putative 'aerosol-generating procedures' is predominantly epidemiological and from the time of the SARS-CoV-1 epidemic in 2003 [4, 5]. Several recent studies have questioned whether these medical procedures should be classified as 'aerosol generating' following quantitation of the aerosol produced during these activities [6-8].

Facemask ventilation is a core airway intervention and listed by the World Health Organization (WHO) as "aerosol generating" [4,5]. The epidemiological evidence for this designation is from a single study that reported an increased risk of SARS-CoV-1 infection from facemask ventilation before tracheal intubation [9]. This risk was determined following interviews with 26 healthcare workers approximately 4 months after contracting SARS-CoV-1 to identify their clinical activity during the period $24 \mathrm{~h}$ before and $4 \mathrm{~h}$ following the performance of tracheal intubation. The authors reported a pooled increased risk of infection after being in the room of a SARS-CoV-1-positive patient during tracheal intubation, where facemask ventilation had been performed (OR 2.8, 95\% Cl 1.3-6.4) [4, 9]. Twenty-two of the 26 healthcare workers were infected by one patient and performing an electrocardiogram was associated with an even higher risk of SARS-CoV-1 infection (OR 3.5, 95\% Cl 1.6-7.9).

No study to date has quantified specifically the aerosol generated during facemask ventilation. Two recent clinical studies, performed in operating theatres, demonstrated relatively little aerosol generation for laryngoscopy and tracheal intubation [6, 10]. The analysis of the phase of facemask ventilation of the anaesthetised patient, before tracheal intubation, demonstrated conflicting results. Brown et al. reported that facemask ventilation was not aerosolgenerating [6]. In contrast, Dhillon et al. recorded an increased particle concentration above background during a period including facemask ventilation [10]. Resolution of these different findings is of crucial importance, as facemask ventilation is a key component of elective and emergency airway management. We therefore co-developed an experimental protocol to test specifically whether facemask ventilation is a high-risk procedure for aerosol generation.
To assess the relative risk, we measured aerosol generation during facemask ventilation and compared this against tidal breathing and volitional coughs, with patients as their own controls.

\section{Methods}

The study protocol was approved by the Greater Manchester Research Ethics Committee as part of the AERATOR study. The methods for aerosol measurement have been described previously [6]. In brief, a prospective environmental monitoring study was conducted in operating theatres in a UK hospital (Southmead Hospital, North Bristol NHS Trust). All recordings were made within operating theatres with an ultraclean ventilation system (EXFLOW 32, Howorth Air Technology, Farnworth, UK) placed in standby mode [11, 12]. This provides an environment with: very low background aerosol concentrations; an air change rate of 25 changes. $^{-1}$ (in line with most other operating theatres in the UK); an air velocity of $0.25 \mathrm{~m} . \mathrm{s}^{-1}$ at $1 \mathrm{~m}$ above the ground; an air temperature of $20^{\circ} \mathrm{C}$; and relative humidity of $40-60 \%$. An optical particle sizer (TSI Incorporated, model 3330, Shoreview, NM, USA) was used to record particle size, concentration and mass (within size range $300 \mathrm{~nm}-10 \mu \mathrm{m}$ in diameter) at a sampling rate of $1 \mathrm{~Hz}$. A 3D-printed funnel was formed of polylactic acid on a RAISE3D Pro2 Printer, (3DGBIRE, Chorley, UK) with $90 \mathrm{~mm}$ height, $10 \mathrm{~mm}$ exit port and maximum diameter of $150 \mathrm{~mm}$. This sampling funnel was connected to the optical particle sizer by a $1.25 \mathrm{~m}$ length of conductive silicone tubing of $4.8 \mathrm{~mm}$ internal diameter. All consented participants were aged over 18 y, ASA physical status 1 or 2 , undergoing routine elective surgery requiring neuromuscular blockade before tracheal intubation and with a negative COVID-19 polymerase chain reaction test in the previous $72 \mathrm{~h}$. Patients with symptomatic gastrooesophageal reflux, a potential or known difficult airway or $\mathrm{BMI} \geq 40 \mathrm{~kg} \cdot \mathrm{m}^{-2}$ were not studied.

The clinical team undertook their normal practice during airway management with the single exception of intentionally generating a leak from the facemask during ventilation attempts after the induction of anaesthesia. The researchers were not involved in the delivery of peri-operative care. All staff in the operating theatre wore airborne personal protective equipment including FFP3-type masks. All participants were supine with head positioning as per the preference of the anaesthetist. An initial period of aerosol sampling was recorded with the patient awake which comprised of $60 \mathrm{~s}$ tidal breathing followed by three volitional coughs, spaced at $30 \mathrm{~s}$ intervals. Sampling was performed 
with the funnel $20 \mathrm{~cm}$ directly above the mouth of the patient. A piece of sampling tubing was cut to $20 \mathrm{~cm}$ and used to guide funnel positioning. The sampling funnel was then directed away from the patient to record background aerosol concentration in the operating theatre while the anaesthetist prepared the patient for induction of anaesthesia.

All patients were pre-oxygenated with an $\mathrm{F}_{1} \mathrm{O}_{2}$ of 1.0. Induction of anaesthesia was performed with titrated intravenous propofol and opioid, followed by rocuronium at a dose of $0.4-0.6 \mathrm{mg} \cdot \mathrm{kg}^{-1}$. Once the patient was unconcious, facemask ventilation was performed with a circle breathing circuit connected to an anaesthetic machine (Aisys $\mathrm{CS}^{2}$, GE Healthcare, Chicago, IL, USA) enabling the measurement of airway pressure and the volume of manual breaths delivered. To ensure standardisation, $60 \mathrm{~s}$ of manual facemask ventilation was performed with a tidal volume of $5-7 \mathrm{ml}^{\mathrm{kg}}{ }^{-1}$ with airway pressures $<20 \mathrm{~cm} \mathrm{H}_{2} \mathrm{O}$ and a respiratory rate of 12-15 breaths. $\min ^{-1}$. Standard anaesthetic monitoring, including waveform capnography and pulse oximetry, was used throughout. No airway adjuncts were required.

If the patient was clinically stable with their lungs easy to ventilate, the anaesthetist relaxed their grip on the mask to create an intentional, audible airway leak at the patientmask interface. The patient's lungs were then ventilated with the intentional leak for a further $60 \mathrm{~s}$. The fresh gas flow and pressure limiting valve were adjusted to ensure the bag refilled to allow manual ventilation during this period. Monitoring of peripheral oxygen saturation was undertaken to ensure it did not fall below 95\%, which would trigger restoration of ventilation with a good seal. During facemask ventilation with an intentional leak, the sampling funnel was positioned towards the side of the facemask towards the leak, maintaining a $20 \mathrm{~cm}$ distance from the mouth of the patient. The funnel was handheld to ensure it could be promptly removed in case of clinical need.

Airway management events were time-stamped by the researcher, including: the period of tidal breathing; coughing; induction of anaesthesia; administration of neuromuscular blockade; start of recording for facemask ventilation with no leak; and start of facemask ventilation with a leak. Facemask ventilation analysis was commenced approximately $60 \mathrm{~s}$ after rocuronium had been administered. Aerosol sampling was continuous throughout the whole period from the induction of anaesthesia to tracheal intubation.

Based on previous work [6], the starting hypothesis was that facemask ventilation would produce no increase in aerosol above background. The sampling methodology was similar to previous work investigating aerosol production during gastro-oesophageal endoscopy [11], where breathing was clearly distinguishable above background. Sample size calculations predicted 10 participants would ensure the study would be adequately powered to detect a difference of clinically important magnitude [13].

Data were processed in the TSI Aerosol Instrument Manager software, and analysed in Origin Pro (Originlab, Northampton, MA, USA) and Prism v9 (Graphpad, San Diego, CA, USA). The normality of data distribution was assessed using the Shapiro-Wilk test. Comparisons were made between aerosol measurements with parametric or non-parametric statistical analyses as appropriate. The significance level was set at $p<0.05$.

\section{Results}

Recordings were made during airway management for 11 patients undergoing elective surgery. There were six women and five men, mean (SD) age 60.0 (18.7) y and BMI 27.1 (5.0) $\mathrm{kg} \cdot \mathrm{m}^{-2}$. The ultraclean ventilation system in the operating theatre environment produced a very low median (IQR [range]) background particle concentration of 2 (1-5 [0-13]) particles. $\left.\right|^{-1}$. Spontaneous quiet tidal breathing was consistently detected above background levels (Fig. 1) with a particle concentration of 191 (77-486 [4-1313]) particles. I $^{-1}$, $\mathrm{p}=0.002$ (Fig. 2a).

The volitional coughs showed a peak aerosol concentration of 1260 (800-3242 [100-3682]) particles. [ $^{-1}$. The analysis of the particle size distributions of these coughs demonstrated the distinctive 'fingerprint' of a cough observed in other studies with the majority of particles (86.5\%) measuring $<1 \mu \mathrm{m}$ in diameter $[6,11,12,14]$. These coughs also had a characteristic temporal profile with a rapid increase in particles which decayed over the subsequent $15 \mathrm{~s}$.

No airway difficulties were experienced and no request was made by the anaesthetists to remove the sampling funnel from the airway management zone. Peripheral oxygen saturation remained $>95 \%$ for all participants during facemask ventilation, including during the period of ventilation with an intentional leak. There were no coughs noted during facemask ventilation with or without a leak. The particle concentration detected during $60 \mathrm{~s}$ of facemask ventilation without a leak was 3 (0-9 [0-43]) particles. ${ }^{-1}$, which was no different to background ( $p=0.43$ ) and much lower than the concentration recorded during tidal breathing ( $p=0.001)$ (Figs. 1 and $2 a$ ).

The particle concentration during facemask ventilation with a leak was 11 (7-26 [1-62]) particles. ${ }^{-1}$, which was approximately five-fold higher than background $(p=0.019)$ 


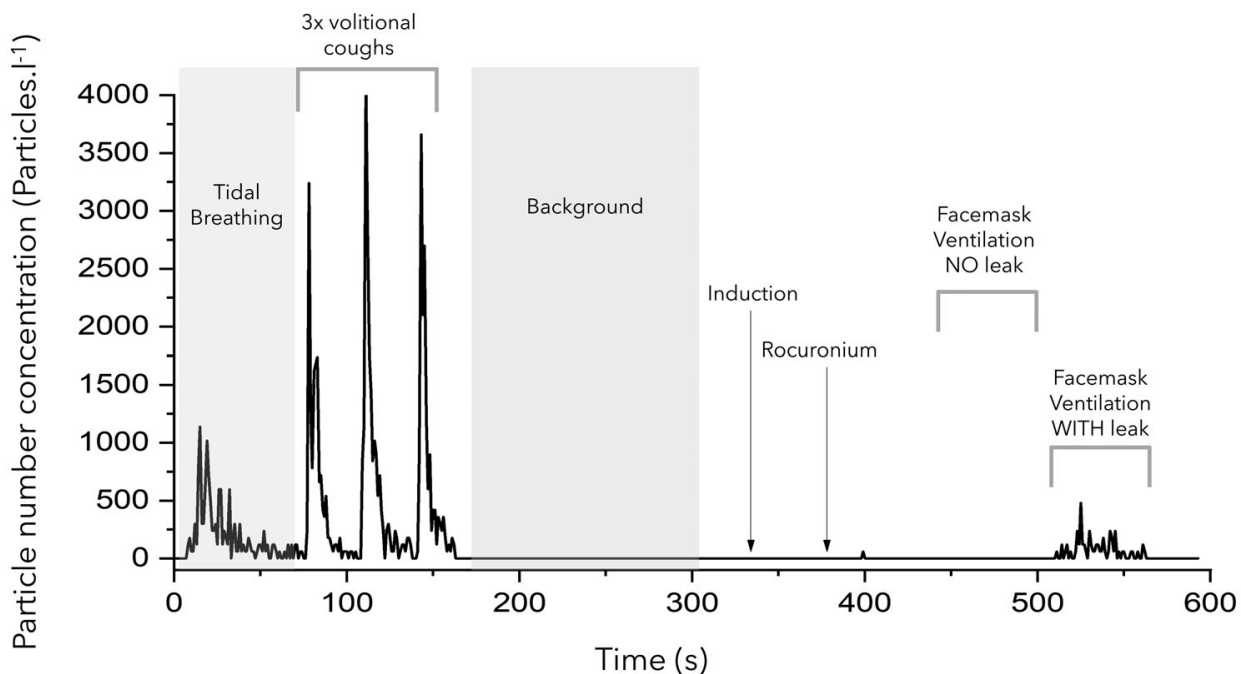

Figure 1 Aerosol concentration measured during the experimental protocol. This shows the concentration of particles detected during baseline respiratory manoeuvres (tidal breathing and voluntary coughs), background monitoring, facemask ventilation with no leak and facemask ventilation with a leak.
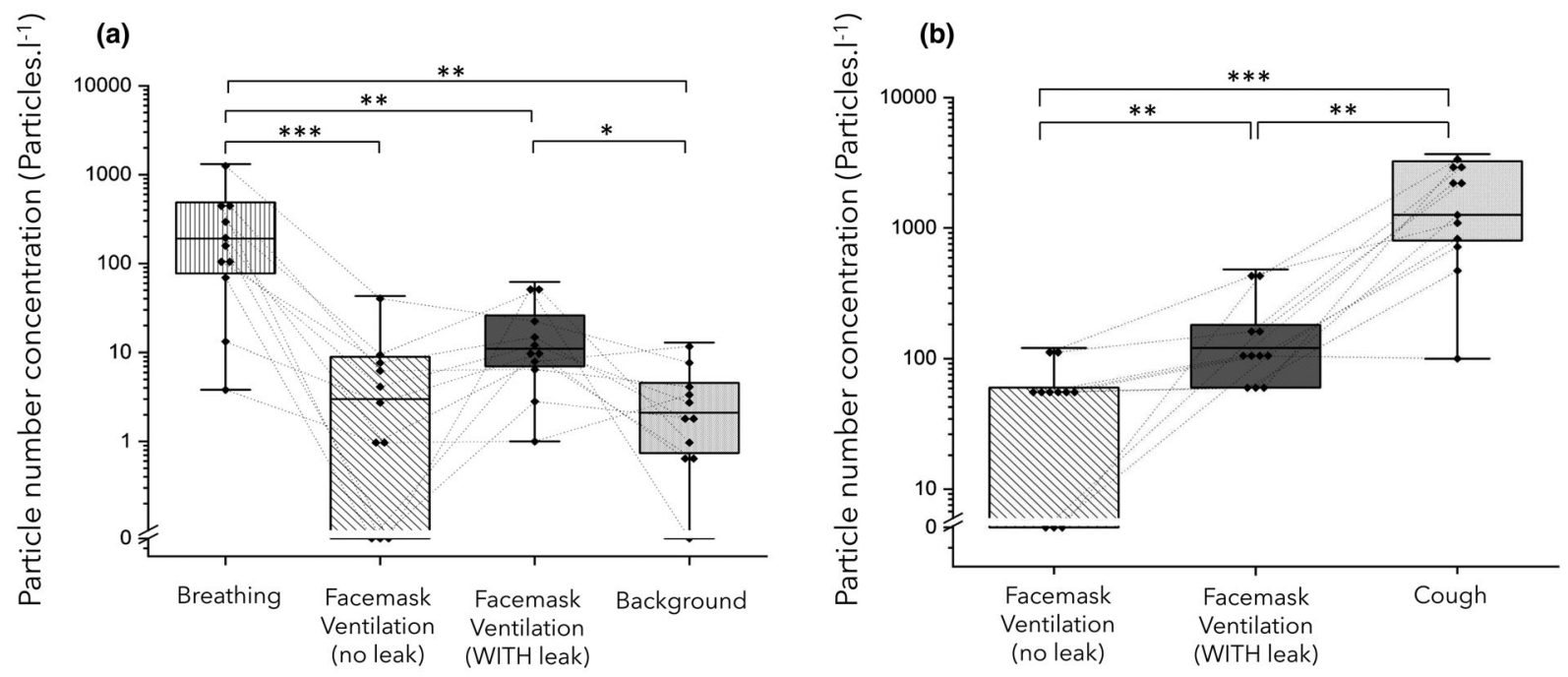

Figure 2 (a) Comparison of particle number concentrations detected during tidal breathing, facemask ventilation with/and without a leak and background levels. (b) Peak particle concentrations from facemask ventilation with/and without leak and cough. Boxes represent IQR, solid horizontal line represents median, Whiskers show range, [ $\bullet$ ] represents individual data points. Dotted lines link values for each participant. Wilcoxon matched pairs, $* * * p \leq 0.001, * * p \leq 0.01, * p \leq 0.05$.

but still much lower (17-fold) than that seen during tidal breathing $(p=0.002)$. The analysis of the difference in particle concentration between facemask ventilation with and without a leak showed no statistically significant difference $(p=0.074)$ (Fig. 2a).

The peak particle concentration recorded during the periods of facemask ventilation without a leak was 60 particles. $\left.\right|^{-1}$ (0-60 [0-120]) as compared with 120 particles..$^{-1}$ (60-180 [60-480]) when there was a leak, which is 20 -fold
( $p=0.002)$ and 10 -fold $(p=0.001)$ lower, respectively, than the particle count detected during a cough (Fig. 2b).

\section{Discussion}

This study demonstrates that facemask ventilation in anaesthetised patients, even with a leak, generates less aerosol than tidal breathing and far less aerosol than a cough. This supports the findings from our previous study which included periods of facemask ventilation as part of 
the intubation sequence [6]. We found no evidence that the procedure of facemask ventilation in these circumstances generates high aerosol concentrations and therefore it should not be classified as an aerosol-generating procedure $[1,15]$. This has implications in a wide range of settings including during routine anaesthetic airway management. The avoidance of facemask ventilation before tracheal intubation or supraglottic airway insertion, due to concerns around aerosol generation, is not supported by this new evidence and likely serves only to increase the risk of encountering difficulties in airway management.

We have used tidal breathing and cough from participants to enable within-subject comparison and relative risk estimation for facemask ventilation. Inter-patient variation was considerable and ranged up to 50 -fold for tidal breathing and 36 -fold during coughing. This is in keeping with previous studies performed by the AERATOR group and others [6, 8 , $11,12,16]$. Therefore, using each participant as their own reference increases the power to generate meaningful comparisons from a relatively small sample. We have also modified our aerosol sampling position to move from $0.5 \mathrm{~m}$ to $0.2 \mathrm{~m}$ so as to be closer to the mouth of the patient. This has increased our ability to detect the emitted aerosol from source and has increased the concentration of particles recorded with tidal breathing and other respiratory activities. There was a 48fold increase in particle concentration detected during tidal breathing when recorded at this closer position (191 vs. 4 particles. $\left.\right|^{-1}$ ) compared with our previous study of supraglottic airway devices performed in the same environment [12]. The higher measured particle concentration closer to the mouth are likely due to decreased particle dispersion and the capture of particles with low momentum in the smaller size range when sampling at $0.2 \mathrm{~m}$ compared with $0.5 \mathrm{~m}$. Despite sampling closer to the source, it is possible some aerosol was not detected during facemask ventilation. However, as the concentration detected was far lower than that produced by tidal breathing, we can infer the relative risk of aerosol generation by facemask ventilation is very low.

The low concentration of aerosol detected during facemask ventilation with an intentional leak is also reassuring given that this represents a worst-case scenario. The particles detected during facemask ventilation with leak likely represent respiratory aerosol originating from the lungs and upper airways during continued positive pressure ventilation rather than from turbulent airflow over the face. This is supported by the fact that these particles were predominantly small - which is consistent with respiratory origin where the smallest particles are thought to be generated [17]. We emphasise, however, that this concentration of aerosol was far lower than the patient would generate if conscious and breathing at rest. We can extend this conclusion further to state that a well-fitting facemask with a good seal reduces emitted aerosol concentration to the point where it is indistinguishable from the near-zero aerosol background, and we have previously demonstrated a well-fitting facemask can prevent bioaerosol leak from a cough [12] by keeping respiratory aerosols within the breathing circuit. This is entirely predictable as the mask forms a physical barrier to aerosol spread.

A limitation of our study is that we intentionally studied a period of facemask ventilation after neuromuscular blockade to focus on the aerosol generation associated with the specific procedure rather than any paroxysmal respiratory event like coughing. However, aerosol sampling was conducted continuously throughout the induction of anaesthesia and the period of facemask ventilation performed immediately before the formally analysed period (i.e. before neuromuscular blockade) did not show increased aerosol concentrations above background (Fig. 1). Previous work performed by our group quantified aerosol generation during facemask ventilation in anaesthetised patients without neuromuscular blockade, which again did not demonstrate aerosol generation [12]. We are confident that our conclusions may be generalisable to the unparalysed patient.

Very low background particle counts, accurate timestamping and a high detector sampling rate are essential for accurate detection and attribution of aerosol from respiratory events and medical procedures. During the conduct of this study, we did not detect any non-attributable spikes of aerosol. We have previously identified a variety of materials present in an operating theatre capable of generating high levels of (non-respiratory) airborne particles, including: patient bedding; gauze; swabs; tube-ties; throat packs; surgical scrubs; and incontinence pads [12]. The study by Dhillon et al., which reported episodes of increased aerosol during the period of facemask ventilation [10], was not performed in an operating theatre with an ultraclean ventilation system, making precise source attribution more challenging. There may also have been other procedures conducted during the induction sequence that could have generated aerosol, such as airway suctioning (currently listed as an aerosol-generating procedure) which also requires further exploration.

In summary, this study demonstrates facemask ventilation, even when performed with an intentional leak, does not generate high levels of bioaerosol. Both tidal breathing and a volitional cough generate many-fold more aerosol than facemask ventilation. On this basis, we believe facemask ventilation should not be considered an aerosol generating procedure. Accumulating evidence demonstrates many procedures currently defined as aerosol 
generating are not intrinsically high risk for generating aerosol, and that natural patient respiratory events often generate far higher levels [6, 7]. Furthermore, some of those procedures that generate aerosol, such as gastroesophageal endoscopy, only do so when the patient coughs [11]. The emerging evidence from quantitative clinical aerosol studies is yet to be incorporated into clinical guidance for aerosol generating procedures and we believe this needs urgent reassessment. Declassification of some of these anaesthesiarelated procedures as aerosol generating would seem appropriate due to their lack of aerosol generation. Our findings also raise the broader question of whether the term 'aerosol generating procedure' is still a useful concept for anaesthetic airway management practice in the prevention of SARS-CoV-2 or other airborne pathogens [18].

\section{Acknowledgements}

The AERATOR study is registered in the ISRCTN registry (N21447815) and is funded by an NIHR-UKRI rapid rolling grant. AS is an NIHR-funded doctoral Research Fellow. BB is supported by the Natural Environment Research Council. $\mathrm{FH}$ is a Wellcome GW4-CAT funded Clinical Doctoral Fellow. AP declares advisory board work for Lateral Pharma and consultancy for and research grants from Eli Lilly for projects unrelated to this study. FM is a co-inventor (and associated patent holder) of a portable isolation hood designed to reduce aerosol exposure from respiratory infections. This report presents independent research commissioned by the National Institute for Health Research (NIHR). The views and opinions expressed by authors in this publication are those of the authors and do not necessarily reflect those of the NHS, the NIHR, UKRI or the Department of Health. No other competing interests declared.

\section{References}

1. Public Health England. COVID-19 infection prevention and control guidance: aerosol generating procedures. 2020. https://www.gov.uk/government/publications/wuhan-novelcoronavirus-infection-prevention-and-control/covid-19-infectionprevention-and-control-guidance-aerosol-generating-procedures (accessed 25/05/2021).

2. World Health Organization. Infection prevention and control of epidemic- and pandemic-prone acute respiratory infections in health care. 2014. https://www.who.int/publications/i/item/ infection-prevention-and-control-of-epidemic-and-pandemicprone-acute-respiratory-infections-in-health-care (accessed 25/ 05/2021).

3. Victoria State Government, Australia. COVID-19 Infection prevention and control guidelines V6.1. 2021. https://www. dhhs.vic.gov.au/covid-19-infection-control-guidelines (accessed 24/07/2021)

4. Tran K, Cimon K, Severn M, Pessoa-Silva CL, Conly J. Aerosol generating procedures and risk of transmission of acute respiratory infections to healthcare workers: a systematic review. PLoS One 2012; 7: e35797.
5. Antimicrobial Resistance and Healthcare Associated Infection Scotland. Assessing the evidence base for medical procedures which create a higher risk of respiratory infection transmission from patient to healthcare worker. 2021. https://hpspubsrepo. blob.core.windows.net/hps-website/nss/3055/documents/1_ agp-sbar.pdf(accessed 11/06/2021).

6. Brown J, Gregson FKA, Shrimpton A, et al. A quantitative evaluation of aerosol generation during tracheal intubation and extubation. Anaesthesia 2021; 76: 174-81.

7. Wilson NM, Marks GB, Eckhardt A, et al. The effect of respiratory activity, non-invasive respiratory support and facemasks on aerosol generation and its relevance to COVID-19. Anaesthesia 2021; 76: 1465-74.

8. Gaeckle NT, Lee J, Park Y, Kreykes G, Evans MD, Hogan CJ Jr. Aerosol generation from the respiratory tract with various modes of oxygen delivery. American Journal of Respiratory and Critical Care Medicine 2020; 202: 1115-24.

9. Raboud J, Shigayeva A, McGeer A, et al. Risk factors for SARS transmission from patients requiring intubation: a multicentre investigation in Toronto, Canada. PLoS One 2010; 5: e10717.

10. Dhillon RS, Rowin WA, Humphries RS, et al. Aerosolisation during tracheal intubation and extubation in an operating theatre setting. Anaesthesia 2021; 76: 182-8.

11. Gregson FKA, Shrimpton AJ, Hamilton F, et al. Identification of the source events for aerosol generation during oesophagogastro-duodenoscopy. Gut 2021. Epub 29 June. https://doi. org/10.1136/gutjnl-2021-324588

12. Shrimpton AJ, Gregson FKA, Brown JM, et al. A quantitative evaluation of aerosol generation during supraglottic airway insertion and removal. Anaesthesia 2021. Epub 20 July. https:// doi.org/10.1111/anae.15542

13. Faul F, Erdfelder E, Buchner A, Lang AG. Statistical power analyses using $G *$ Power 3.1: tests for correlation and regression analyses. Behaviour Research Methods 2009; 41: 1149-60.

14. Gregson FKA, Watson NA, Orton CM, et al. Comparing aerosol concentrations and particle size distributions generated by singing, speaking and breathing. Aerosol Science and Technology 2021; 55: 681-91.

15. Centers for Disease Control and Prevention. Which procedures are considered aerosol generating procedures in healthcare settings. Healthcare Workers FAQs. 2020. https://www.cdc.gov/ coronavirus/2019-ncov/hcp/faq.html?CDC_AA_refVal=https\%3A \%2F\%2Fwww.cdc.gov\%2Fcoronavirus\%2F2019-ncov\%2Fhcp\% 2Finfection-control-faq.html (accessed 25/05/2021).

16. Asadi S, Wexler AS, Cappa CD, Barreda S, Bouvier NM, Ristenpart WD. Aerosol emission and superemission during human speech increase with voice loudness. Scientific Reports 2019; 9: 2348.

17. Johnson GR, Morawska L, Ristovski ZD, et al. Modality of human expired aerosol size distributions. Journal of Aerosol Science 2011; 42: 839-51.

18. Hamilton F, Arnold D, Bzdek BR, et al. Aerosol generating procedures: are they of relevance for transmission of SARSCoV-2? Lancet Respiratory Medicine 2021; 9: 687-9.

\section{Appendix 1}

The Aerosolisation and transmission of SARS-CoV-2 in healthcare settings (AERATOR) study group are also authors of this study:

C. White, J. Murray, D. Arnold, G. Nava, N. Maskell, J. Dodd, E. Moran (Southmead Hospital, North Bristol NHS Trust); J. Keller, M. Gormley (University Hospitals Bristol and Weston NHS Trust); S. Sheikh, A. Davidson (University of Bristol). 


\section{University Library}

\section{- M I N E R VA}

\section{A gateway to Melbourne's research publications}

Minerva Access is the Institutional Repository of The University of Melbourne

\section{Author/s:}

Shrimpton, AJ;Brown, JM;Gregson, FKA;Cook, TM;Scott, DA;McGain, F;Humphries, RS;Dhillon, RS;Reid, JP;Hamilton, F;Bzdek, BR;Pickering, AE

Title:

Quantitative evaluation of aerosol generation during manual facemask ventilation

Date:

2021-10-26

\section{Citation:}

Shrimpton, A. J., Brown, J. M., Gregson, F. K. A., Cook, T. M., Scott, D. A., McGain, F., Humphries, R. S., Dhillon, R. S., Reid, J. P., Hamilton, F., Bzdek, B. R. \& Pickering, A. E. (2021). Quantitative evaluation of aerosol generation during manual facemask ventilation. ANAESTHESIA, 77 (1), pp.22-27. https://doi.org/10.1111/anae.15599.

Persistent Link:

http://hdl.handle.net/11343/296637

License:

CC BY 